\title{
A Proton Reaction Cross Sections Measured in the BNL/AGS E943 Experiment
}

F. S. Dietrich, E. P. Hartouni, G. J. Schmid, R. Soltz, W. P. Abfalterer, R. C. Haight, L. S. Waters, A. L. Hanson, R. W. Finlay, G. S. Blanpied

Nuclear Applications of Accelerator Technology (AccApp00), Washington, D. C., November 12-15, 2000

November 28, 2000 


\section{Disclaimer}

This document was prepared as an account of work sponsored by an agency of the United States Government. Neither the United States Government nor the University of California nor any of their employees, makes any warranty, express or implied, or assumes any legal liability or responsibility for the accuracy, completeness, or usefulness of any information, apparatus, product, or process disclosed, or represents that its use would not infringe privately owned rights. Reference herein to any specific commercial product, process, or service by trade name, trademark, manufacturer, or otherwise, does not necessarily constitute or imply its endorsement, recommendation, or favoring by the United States Government or the University of California. The views and opinions of authors expressed herein do not necessarily state or reflect those of the United States Government or the University of California, and shall not be used for advertising or product endorsement purposes.

This work was performed under the auspices of the U.S. Department of Energy by University of California, Lawrence Livermore National Laboratory under Contract W-7405-Eng-48. 


\section{PROTON REACTION CROSS SECTIONS MEASURED IN THE BNL/AGS E943 EXPERIMENT}

\author{
F. S. Dietrich, E. P. Hartouni, \\ G. J. Schmid, R. Soltz \\ Lawrence Livermore National Laboratory \\ L-050, P. O. Box 808
}

Livermore, CA 94550

(925) 422-4521

\author{
W. P. Abfalterer, R. C. Haight, \\ L. S. Waters \\ Los Alamos National Laboratory \\ Los Alamos, NM 87545 \\ (505) 665-4127
}

\author{
A. L. Hanson \\ Brookhaven National Laboratory \\ P. O. Box 5000 \\ Upton, NY 11973 \\ (631) 344-3499
}

\author{
R. W. Finlay \\ Department of Physics and Astronomy \\ Ohio University \\ Athens, OH 45701 \\ (740) 593-1974
}

\author{
G. S. Blanpied \\ Department of Physics and Astronomy \\ University of South Carolina \\ Columbia, SC 29208 \\ (803) 777-2599
}

\begin{abstract}
We have measured proton reaction cross sections over a wide mass and energy range at the Brookhaven AGS accelerator. The samples were elemental $\mathrm{Be}, \mathrm{C}, \mathrm{Al}$, $\mathrm{Cu}, \mathrm{W}$, and $\mathrm{Pb}$; the measurements were carried out at ten incident proton kinetic energies in the range 0.54 to 7.8 $\mathrm{GeV}$. The experiment was similar to an earlier experiment in the $200-550 \mathrm{MeV}$ range by Renberg et $a l .{ }^{1}$ The new results are in good agreement with those of Renberg et al. at the overlap point near $550 \mathrm{MeV}$. The combined results of the two experiments show an energy dependence expected from the behavior of the nucleonnucleon cross sections. The results are reproduced by calculations based on variants of the impulse approximation and Glauber theory.
\end{abstract}

\section{INTRODUCTION}

Codes such as MCNPX rely for their accuracy on well-measured (or calculated) cross section data. Among the most important cross sections are neutron total and proton reaction cross sections. These data are used directly in the codes, where they provide a sum on all reaction processes, and also indirectly through their use in the development of models (such as the optical model) that are used for the calculation of other reactions.

Very accurate $(1 \%)$ measurements of neutron total cross sections in the $5-560 \mathrm{MeV}$ range were recently carried out at LANSCE/WNR under the auspices of the Accelerator Production of Tritium (APT) project ${ }^{2}$. Since neutron measurements of such accuracy are very difficult above this energy range, we have undertaken proton reaction cross section measurements in the energy range $0.54-7.8 \mathrm{GeV}$. Proton reaction cross sections represent the sum of all possible reaction processes on a nucleus with the exception of elastic scattering. The measurements were carried out in the B1 beam line at the Brookhaven AGS accelerator as experiment E943. Measurements were made on elemental samples of $\mathrm{Be}, \mathrm{C}, \mathrm{Al}, \mathrm{Cu}, \mathrm{W}$, and $\mathrm{Pb}$. The accuracy achieved was approximately $3 \%$. The results were used to test theoretical models for microscopic optical potentials based on the impulse approximation, a modified impulse approximation that accounts for nuclear-medium modification of the nucleon-nucleon interaction, and Glauber theory. The good agreement obtained with these models suggests that they are useful for calculating reaction cross sections where measurements have not been made.

\section{EXPERIMENTAL DETAILS}

The measurements were carried out by a transmission technique, in which the beam of protons was incident on a sample, and the transmitted protons (as well as reaction products) were detected in a coaxial array of six circular plastic-scintillator counters mounted downstream of the sample. The diameters of these counters ranged from 10.6 to $45 \mathrm{~cm}$. The count rates in the detectors with the sample in and out of the beam were compared to obtain the sample transmission. The experimental arrangement and the analysis of the data were very similar to those in Ref. 1 .

Particle identification was necessary, since the proton beam in the AGS B1 beam line is a mixture of electrons, muons, pions, kaons, and protons at a momentum selected by analyzing magnets upstream of the experiment. This was accomplished by time of flight between two $3-\mathrm{cm}$ diameter beam counters separated by approximately $10 \mathrm{~m}$ upstream of the sample. Above $4 \mathrm{GeV} / \mathrm{c}$ momentum, time of flight identification was insufficient, and for these momenta a Cerenkov counter was used to separate out the 
protons. The two beam counters were also used to trigger the data taking electronics. When a coincidence between these counters was obtained, the pulse height and flight time (relative to the downstream beam counter) were recorded for all counters in the experiment.

The six samples were mounted on a wheel that rotated to a new position every 40 seconds. Two of the positions on the 8-position wheel were empty, in order to make the sample-out measurements. A second wheel placed upstream of the samples contained two polyethylene energy degraders that were positioned in the beam during the sample-out measurements. This ensured that the energy of the beam striking the main counters was approximately the same during the sample-in and sampleout conditions. Thicknesses of the samples were chosen so that their transmission was approximately $90 \%$.

The six counters in the main counter array subtended six different solid angles as seen from the samples. The counters were mounted on cart that could be moved along the beam axis. At each energy, the cart was placed at two different positions to allow data taking at a total of twelve solid angles. The detector positions were chosen so that approximately the same range of elastic-scattering momentum transfer was encompassed at each energy.

In analyzing the data, cuts were made on the time of flight between the two beam counters to select incident protons. Analysis of the Cerenkov data to achieve particle separation at the higher momenta is currently in progress. Cuts were also made on the pulse height in all counters to ensure that low-energy pulses corresponding to Cerenkov radiation in the light guides leading from the circular counters to the phototubes was eliminated.

As was done in Ref. 1, a partial cross section at each solid angle was calculated from the measured sample-in to sample-out count-rate ratio. A correction was made for elastic scattering events that missed each counter, since the experimental technique assumes that elastically scattered protons are detected and therefore do not contribute to the measured attenuation in the samples. These corrections were made using elastic angular distributions calculated by Glauber theory ${ }^{3}$. Only counters for which this correction was smaller than $10 \%$ were included in the further analysis. The final value of the cross section was obtained by extrapolating the partial cross sections to zero solid angle. This extrapolation is needed to account for the fact that debris from a nuclear reaction in the sample may be detected in the counters, since such events will not contribute to the apparent sample attenuation. Fig. 1 shows an example of the behavior of the partial cross sections as a function of solid angle for the case of $1380 \mathrm{MeV}$ protons incident on the $\mathrm{Al}$ sample.

Fig. 2 shows preliminary results for proton reaction cross sections on $\mathrm{Al}$ at the energies for which time-of-flight particle identification was sufficient. For these preliminary results the zero-degree extrapolations were made by eye. Combined statistical and systematic errors are estimated at $3 \%$. The results are seen to be in good agreement with the experiment of Ref. 1 at the point of overlap near $540 \mathrm{MeV}$.

The combination of the present results and those of Ref. 1 clearly shows a rise in the reaction cross section in the region $500-1000 \mathrm{MeV}$. This behavior of the protonnucleus cross section mirrors the energy dependence of the elementary nucleon-nucleon cross sections, shown in Fig. 3 for the proton-proton total cross section.

\section{CALCULATIONS}

The data in the present experiment and in Ref. 1 were used to test formulations of the microscopic optical potential. In microscopic optical potentials, the nucleonnucleus potential is obtained by convolution of the nuclear density with an effective nucleon-nucleon interaction. The potential is used in a relativistically-modified Schrodinger equation to yield observables such as the neutron total and proton reaction cross sections. In the present work proton densities were taken from elastic scattering results as tabulated in Ref. 4, together with a reasonable assumption for the neutron densities. Results of such calculations for neutron total cross sections and the proton reaction cross sections of Ref. 1 are in the course of publication ${ }^{2}$.

The dotted curve in Fig. 2 shows an impulseapproximation calculation based on the Franey-Love ${ }^{5}$ effective interaction, which has been tabulated between 50 and $1000 \mathrm{MeV}$. It is in reasonable agreement with the data in the $400-1000 \mathrm{MeV}$ region, but disagrees at lower energies. This deficiency is well understood to be due to the need for modifications of the effective nucleon-nucleon interaction in the nuclear medium. An empirical effective interaction in the $135-650 \mathrm{MeV}$ range involving densitydependent modifications of the Franey-Love interaction has been reported by Kelly and Wallace ${ }^{6}$. Calculations based on this interaction, shown by the solid curve in Fig. 2 , agree well with the data. Glauber theory ${ }^{3}$ calculations avoid explicit use of a wave equation by using an eikonal approximation, and employ a simple description of the nucleon-nucleon interaction based directly on cross sections such as those shown in Fig. 3. Results of Glauber calculations, shown by the dot-dash curve in Fig. 2, are seen to provide a good description of the data at high energies where effective interactions based on the impulse approximation are not available. 


\section{SUMMARY AND CONCLUSIONS}

We have measured proton reaction cross sections on a set of samples spanning the periodic table at energies ranging from 0.54 to $7.8 \mathrm{GeV}$. The experimental arrangement was similar to that of Renberg et al. ${ }^{1}$, who measured reaction cross sections in the $200-550 \mathrm{MeV}$ range. The excellent agreement between the two experiments at the matching energy near $540 \mathrm{MeV}$ suggests that the two sets of measurements may be used to delineate the energy dependence of proton reaction cross sections in the $500-1000 \mathrm{MeV}$ region where the underlying nucleon-nucleon cross sections are varying rapidly. The energy dependence is well reproduced by calculations based on a modified impulse approximation and by Glauber theory, as illustrated by preliminary results on an aluminum sample. This result, together with a similarly successful reproduction of neutron total cross sections ${ }^{2}$, indicates that these models may be useful for quantitative prediction of proton and neutron cross sections where data are lacking.

\section{ACKNOWLEDGMENTS}

We are grateful to the Accelerator Production of Tritium and Proton Radiography projects for their sponsorship of this work. We are indebted to Richard Hammer (Los Alamos) and the late Dennis Chakedis (Livermore) for their essential contributions to the success of these measurements, as well as to the Brookhaven AGS staff. The work was performed by the USDOE under contracts W-7405-ENG-48 (Livermore), W-7405-ENG-36 (Los Alamos), and DE-AC02-98CH10886 (Brookhaven).

\section{REFERENCES}

1. P. U. Renberg et al., Nucl. Phys. A183, 81 (1972).

2. W. P. Abfalterer et al., accepted for publication in Phys. Rev. C (2001); see also W. P. Abfalterer et al., LANL Report LA-UR-99-666 (February, 1999).

3. R. J. Glauber, "High Energy Collision Theory," in Lectures in Theoretical Physics, Vol. 1, p. 315, Interscience Publishers, New York, 1959.

4. H. de Vries, C. W. de Jager, and C. de Vries, At. Data and Nucl. Data Tables 36, 495 (1987).

5. M. A. Franey and W. G. Love, Phys. Rev. C 31, 488 (1985).

6. J. J. Kelly and S. J. Wallace, Phys. Rev. C 49, 1315 (1994).

\section{Al, $1380 \mathrm{MeV}$}

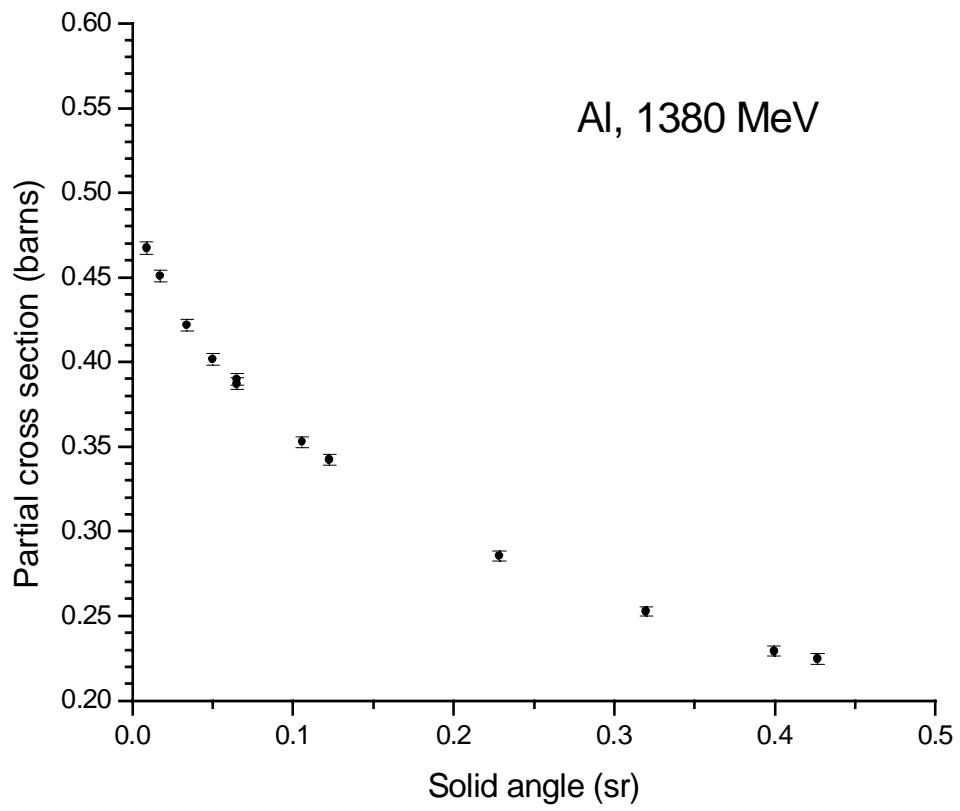

Figure 1. Example (for 1380-MeV protons on the Al sample) of the partial cross sections as a function of solid angle after the elastic-scattering correction. The final cross section is obtained by extrapolating to zero solid angle. 


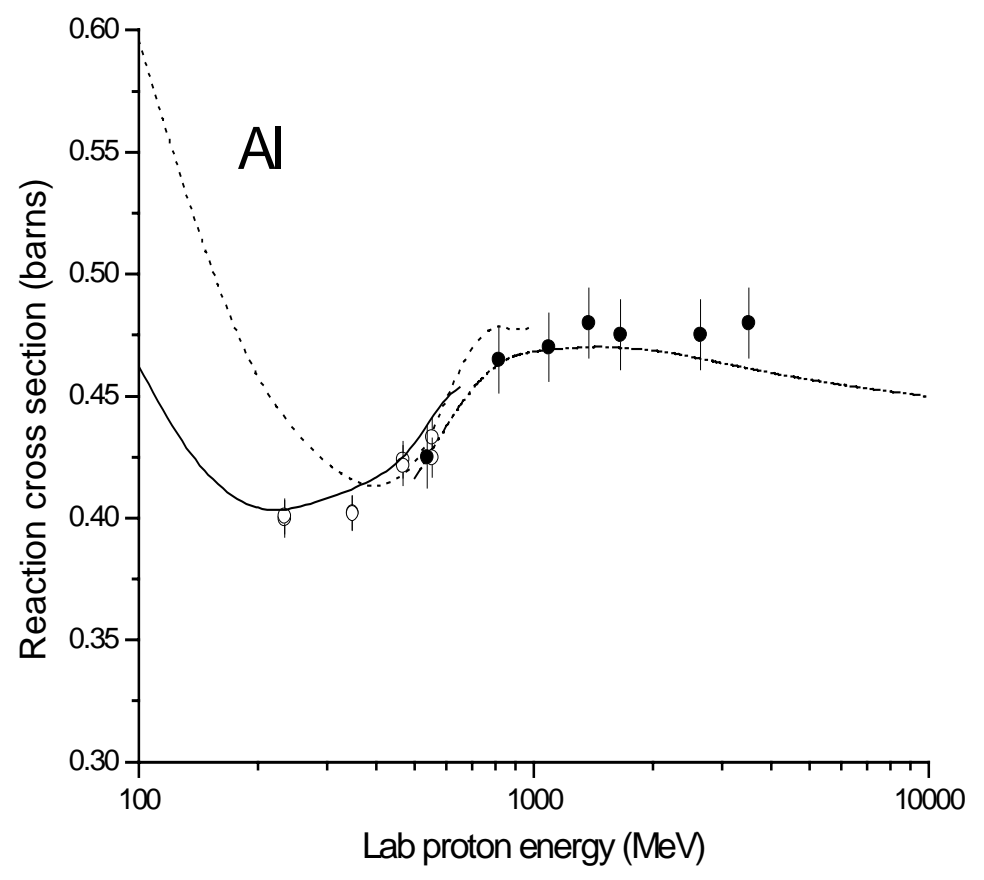

Figure 2. Preliminary results of the present measurements for aluminum are shown by closed circles; those of Ref. 1 by open circles. The calculated curves were made with the Glauber theory (dot-dash), impulse approximation (dotted), and a nuclear-medium modified impulse approximation (solid); see text.

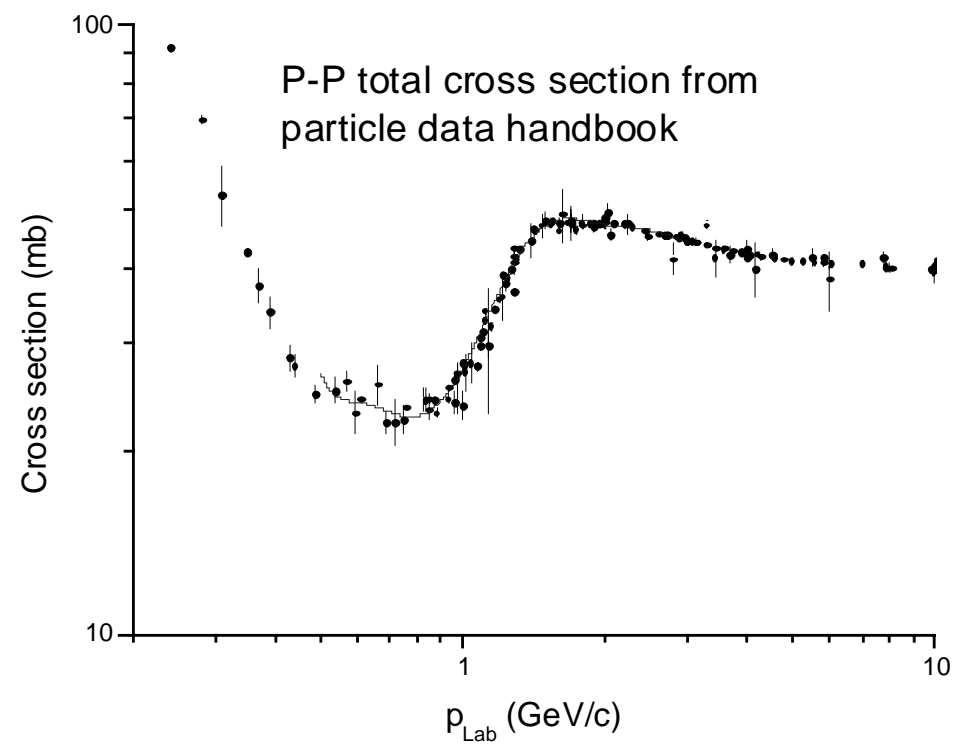

Figure 3. Proton-proton total cross sections. The rise in the 1-2 GeV/c region is mirrored in the data and calculations shown in Fig. 2. The solid curve is a polynomial fit used in the Glauber calculations. Note the horizontal axis is labeled by momentum, while that in Fig. 2 is labeled by kinetic energy. 